\title{
ERRATUM
}

\section{Erratum: Long-range self-organization of cytoskeletal myosin II filament stacks}

\section{Shiqiong Hu, Kinjal Dasbiswas, Zhenhuan Guo, Yee-Han Tee, Visalatchi Thiagarajan, Pascal Hersen, Teng-Leong Chew, Samuel A. Safran, Ronen Zaidel-Bar and Alexander D. Bershadsky}

Nature Cell Biology 19, 133-141 (2017); published online 23 January 2017; corrected after print 31 January 2017

In the version of this Letter originally published, the numbering of Supplementary Video files and the references to those files in the text did not match. All online versions of the Letter have been corrected so that the Supplementary Videos are numbered sequentially from 1-17. 